\title{
Prevalence of Neonatal Sepsis and Associated Factors among Neonates in Neonatal Intensive Care Unit at Selected Governmental Hospitals in Shashemene Town, Oromia Regional State, Ethiopia, 2017
}

\author{
Aytenew Getabelew, ${ }^{1}$ Mihret Aman, ${ }^{1}$ Endashaw Fantaye, ${ }^{1}$ and Tomas Yeheyis $\mathbb{D}^{2}$ \\ ${ }^{1}$ Department of Nursing, College of Medicine and Health Science, Ambo University, Ambo, Ethiopia \\ ${ }^{2}$ Department of Nursing, College of Medicine and Health Science, Arba Minch University, Arbaminch, Ethiopia
}

Correspondence should be addressed to Tomas Yeheyis; tyeheyis@gmail.com

Received 24 April 2018; Revised 25 June 2018; Accepted 12 July 2018; Published 2 August 2018

Academic Editor: Lavjay Butani

Copyright (C) 2018 Aytenew Getabelew et al. This is an open access article distributed under the Creative Commons Attribution License, which permits unrestricted use, distribution, and reproduction in any medium, provided the original work is properly cited.

\begin{abstract}
Background. Neonatal sepsis is an important cause of morbidity and mortality among neonates in developing countries accounting for $30-50 \%$ of total deaths each year. Childhood mortality is often used as broad indicator of the social development or a specific indicator of health conditions of a country. Objectives. The objective of this study is to assess the prevalence of neonatal sepsis and associated factors among neonates admitted in neonatal intensive care unit at two hospitals in Shashemene town, Ethiopia. Method. An institution based cross-sectional study with retrospective document review method was conducted in NICUs of two governmental hospitals in Shashemene town. Sample size was calculated by using single population proportion sample formula and the final sample size was 244 . The study subject was selected by using systematic random sampling method, and adopted data collection tool was used. Then the collected data was coded and entered in to SPSS for windows version 20.0 for cleaning, editing, and analysis. Binary and multiple logistic regressions have been used to observe the association between independent variables and dependent variable. Result. The overall prevalence of neonatal sepsis in this study was $77.9 \%$. From this $65 \%$ and $35 \%$ of neonates developed early onset neonatal sepsis and late onset neonatal sepsis, respectively. This study found out that age of neonates, birth asphyxia, and use of oxygen via mask were significantly associated with neonatal sepsis. Conclusion and Recommendation. The most risk factors of neonatal sepsis were identified as age of neonates, birth asphyxia, and use of oxygen via mask strongly associated with prevalence of NS. Based on this results we recommend the concerned body to focus on the prevention of risk factors rather than treating the disease after it occurs.
\end{abstract}

\section{Background}

Deaths occurring in the neonatal period each year account for $41 \%$ (3.6 million) of all deaths in children under 5 years. The majority of these deaths occur in low income countries and almost 1 million of these deaths are attributable to infectious causes including neonatal sepsis, meningitis, and pneumonia. Furthermore, neonatal mortality for different African countries ranges from 68 per 1000 live births in Liberia to 11 per 1000 live births in South Africa [1].

Neonatal sepsis contributes substantially to neonatal morbidity and mortality and is an ongoing major global public health challenge [2]. According to the World Health Organization (WHO), four million newborn children die each year during the first four weeks of their lives. Of these, $75 \%$ die prematurely during the first week of life $[3,4]$. The major causes of neonatal deaths globally were estimated to be infections (35\%), preterm births (28\%), intrapartum related complication (24\%), and asphyxia (23\%). Sepsis is the commonest cause of neonatal mortality and is probably responsible for $30-50 \%$ of the total neonatal deaths each year in developing countries [5].

According to the current united nation estimate, the neonatal death reduced by $48 \%$ from the 1990 estimate to 28 
per 1000 live births in 2013 while the reduction rate of underfive mortality rate was about $67 \%$ [6]. The16 EDHS reported that neonatal mortality rate is $29 / 1000$ live birth, which has a reduction from the 2005 EDHS report of 39/1000 live births and 2011EDHS report of 37/1000 live birth.

The common causes of neonatal mortality in Ethiopia are infection, asphyxia, and preterm birth [7]. Many women do not generally seek formal healthcare during pregnancy, child birth, and puerperal. This has a major impact on care seeking for and survival of the new born. Less than third of women receive antenatal care and $90 \%$ are assisted by unskilled attendants: TBAs (26\%) relatives (58\%) or alone (6\%). Almost no one (3.5\%) receives postnatal care [8].

Newborn survival is an issue of great concern to the world and especially to developing countries. Care for the neonate often receives little attention in maternal and child health programs. Though various efforts have been made by Ethiopian government to reduce neonatal mortality some studies in Gondar, Black Lion specialized hospital and Bishoftu general hospital, show that it is still high. So it is important to do additional research regarding this title typically on associated factors.

Therefore, this research was conducted to find out the prevalence of neonatal sepsis and associated factors in Shashemene town at two governmental hospitals, South Ethiopia. In addition it will provide opportunity for stake holders to reduce the problem by working on identified factors.

\section{Methods and Materials}

2.1. Study Setting, Design, Period. The study is conducted in Shashemene town, Oromia Regional State, South Ethiopia, at two governmental hospitals. Shashemene referral hospital which is found in Shashemene town situated $238 \mathrm{KM}$ from Addis Ababa and 9 KM North from Shashemene town and Melkaoda general hospital which is located in near Shashemene town, $250 \mathrm{~km}$ away from the capital city, Addis Ababa. This study was carried out using institution based cross section study with retrospective document review from February 5, 2017 to February 30, 2017.

2.2. Sample Size Determination and Sampling Technique. The sample size was determined by using single population proportion formula and the proportion was taken from the previous literature in Ethiopia. According to study conducted at Black Lion specialized hospital, the prevalence of neonatal sepsis was $44.7 \%$ [9].

By considering 95\% confidence interval (CI) and 5\% marginal error the, sample size was calculated as follows:

$$
\text { Where } \mathbf{n}=\left(Z_{\partial / 2}\right)^{2} \frac{\mathbf{p}(1-p)}{\mathbf{d}^{2}}
$$

$\mathrm{n}=$ required sample size

$\mathrm{Z}=$ the standard normal deviation at $95 \%$ confidence interval; $=1.96$
$\mathrm{P}=$ proportion of neonatal sepsis among neonates admitted in NICU with prevalence of 44.7

$\mathrm{d}=$ margin of error that can be tolerated, $5 \%(0.05)$

$1-p=$ proportion of population that do not possess the character of interest.

Therefore,

$$
\begin{aligned}
\mathbf{n} & =\left(Z_{\partial / 2}\right)^{2} \frac{\mathbf{p}(1-p)}{\mathbf{d}^{2}}=\frac{(1.96)^{2}(0.447)(0.553)}{(0.05)^{2}} \\
& =380
\end{aligned}
$$

By considering nonresponse rate $5 \%$, our total sample size was 399 neonates.

Total number of our study population was less than 10,000 , which means that the total number of neonates admitted to this two hospital within one year was 626 , so correction formula was used; the final sample size was $\mathbf{2 4 4}$ neonates.

The study populations was neonate's card who were admitted and treated in NICUs of two government hospitals in the area. These two hospitals are selected purposively because these are the only hospitals in the area which have NICU and render service in this arena. The number of study subjects for each hospital was allocated proportionally after identifying the number of admissions in each hospital in the last one year. Study Subjects were selected by using systematic random sampling method (every Kth) after calculating the "Kth" value by dividing the total number of neonates within the last one year to the required sample size which was allocated proportionally for each hospital and the first subject is selected by lottery method. Medical records with full information were used and if the chart is missing the next in the queue was used (Figure 1).

In this study neonatal is asserted when a medical diagnosis of the neonate is stated as 'neonatal sepsis' by the physician in the neonate's medical record chart.

2.3. Instrument and Data Collection Technique. The data was collected using checklist prepared by reviewing different literatures; the check list contains contain three parts: sociodemographic characteristics; maternal information; and part neonatal information for neonatal sepsis. Records of neonates for the last one year (from February 1/ 2016 to February1/2017) were reviewed. This involves going through log book records of neonates with the diagnosis of sepsis between the times from 2016 to 2017. The medical files were traced using the patient card numbers on the log book registry. If there was incomplete maternal information on the neonatal card, the maternal card was traced by using neonatal card number.

2.4. Data Analysis. After the data was collected, questionnaires were reviewed and organized by investigators. The data were entered after defining variables and analyzed using SPSS v. 20.0 statistical software. Binary Logistic regression was performed. COR (crude odds ratio) along with $95 \%$ confidence interval was used to determine the existence of an 


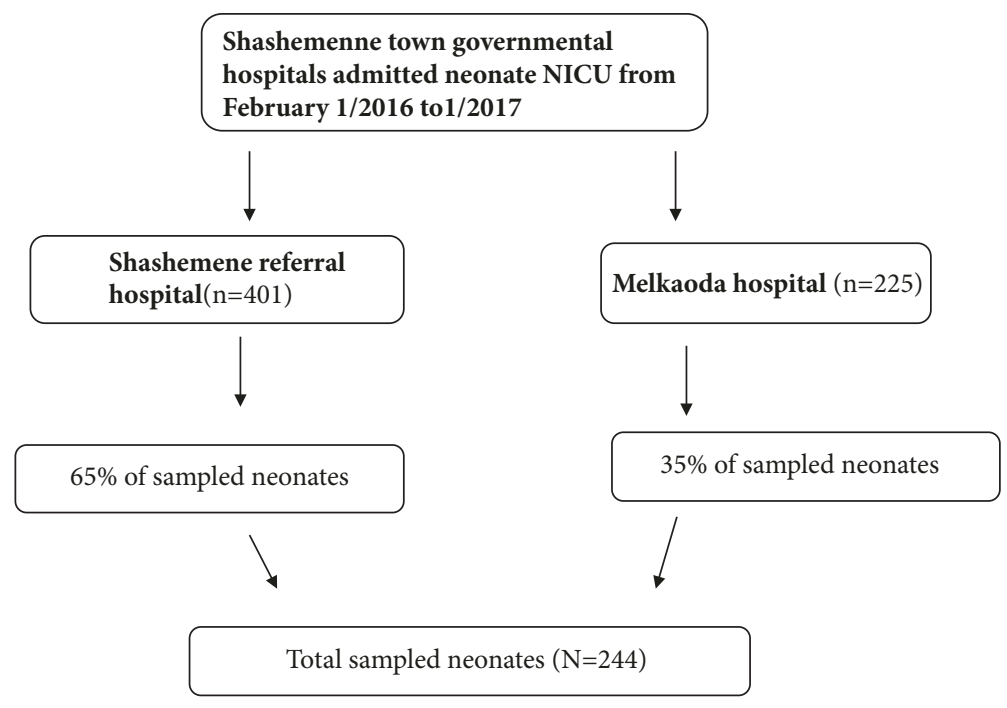

FIGURE 1: Schematic representation of sampling procedure of the study.

association between independent and dependent variables. Then multivariate logistic regression was used to decrease the effect of confounding factors. Statistical significance was declared with $\mathrm{p}$ value less than 0.05 for multivariable and 0.25 for bivariate logistic regressions. Finally, the result is presented using tables, texts, and other pictorial representation.

2.5. Data Quality Control. Before the data collection, the data collectors made sure that they have a common understanding of the questionnaire and the questionnaire was translated to Amharic and back to English. After data collection, internal consistency was checked by cross-checking the collected data within on every day of data collection.

2.6. Ethical Consideration. Ethical clearance was sought from Ambo University, College of Health Science and Department of Nursing Ethical Review Committee. After explaining the purpose and the possible benefit of the study, permission to gather data was obtained from the medical directors of respective hospitals and heads of the neonatal intensive care unit. The respondent's privacy and confidentiality of the information were assured throughout the study procedure.

\section{Results}

3.1. Sociodemographic Characteristics. Among 244 reviewed cards of neonates, 181 (74\%) of them were aged between 0 and 7 days while 63 (26\%) of neonates were aged between 8 and 28 days. Among neonates who enrolled in this study more than half of them were male $142(58.2 \%)$ and the rest are females. Majority (37.7\%) of the mothers were aged between 20 and 24 years (Table 1).

3.2. Prevalence of Sepsis and Maternal Risk Factors for Neonatal Sepsis. Among 244 neonates who were admitted in NICU $190(77.9 \%)$ had neonatal sepsis (Figure 2).

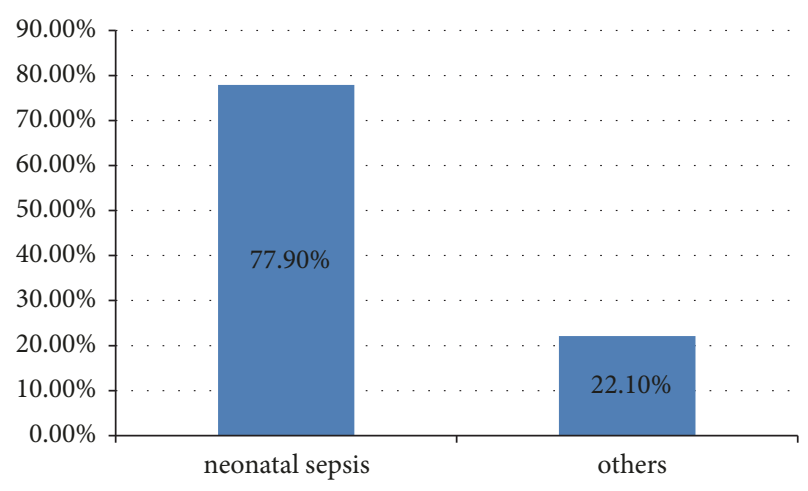

Figure 2: Diagnosis of neonatal sepsis and others cases, in Shashemene referral hospital and Melkaoda General Hospital.

From this were $123(64.7 \%)$ who had early onset neonatal sepsis and 67 (35.3\%) who had late onset neonatal sepsis. Among 244 sampled neonates 22 (9\%) of their mothers had history of UTI and among this 14 (5.7\%) neonates had developed neonatal sepsis. Among a total of sampled neonates $17(7 \%)$ their mothers had history of meconium stained amniotic fluid. Out of this 10 (4\%) of them developed neonatal sepsis. Concerning place of delivery 181 (75\%) of neonates were delivered in hospital and, out of this, 144 (79.6\%) neonates developed neonatal sepsis. About 36 (14.6\%) were delivered in health center; from this $27(11 \%)$ of neonates had neonatal sepsis (Table 2).

3.3. Neonates Risk Factors for Neonatal Sepsis. Among the total neonates 155 (63.5\%) neonates had normal birth weight and $62(25 \%)$ were of low birth weight. Out of normal birth weight and low birth neonates about $52.9 \%$ and $17.6 \%$ neonates had developed neonatal sepsis, respectively. Among the total 244 neonates $65(26.6 \%)$ neonates were reported to have gestational age less than 37 weeks and 42 (17.2\%) of them 
TABLE 1: Sociodemographic characteristics of neonates and mothers unit at selected governmental hospital in Shashemene town, Oromia regional state, south ethiopia, 2017G.

\begin{tabular}{|c|c|c|c|c|c|}
\hline \multirow{2}{*}{ Address } & & \multirow{2}{*}{ Frequency } & \multicolumn{3}{|c|}{ sepsis } \\
\hline & & & Early(\%) & Late onset & Others \\
\hline Shashemenne & & $121(49.6 \%)$ & $58(23.7 \%)$ & $33(13.5 \%)$ & $30(12.2 \%)$ \\
\hline Others & & $123(50.4 \%)$ & $65(26.6 \%)$ & $34(13.9 \%)$ & $24(9.8 \%)$ \\
\hline \multirow{2}{*}{ Age of neonates } & 0-7 day & $181(74 \%)$ & $134(55 \%)$ & - & $47(19 \%)$ \\
\hline & 8-28 days & $63(26 \%)$ & - & $56(23 \%)$ & $7(3 \%)$ \\
\hline \multirow{2}{*}{ Sex of neonate } & Male & $142(58.1 \%)$ & $75(30.7 \%)$ & $35(14.3 \%)$ & $32(13.1 \%)$ \\
\hline & Female & $102(41.8 \%)$ & $48(19.6 \%)$ & $32(13.1 \%)$ & $22(9.1 \%)$ \\
\hline \multirow{5}{*}{ Maternal age } & $<19$ & $42(17.2 \%)$ & $21(8.6 \%)$ & $12(4.9 \%)$ & $9(3.7 \%)$ \\
\hline & $20-24$ & $92(37.7 \%)$ & $51(20.9 \%)$ & $26(10.6 \%)$ & $15(6.2 \%)$ \\
\hline & $25-29$ & $75(30.7)$ & $39(15.9 \%)$ & $17(6.9 \%)$ & $19(7.7 \%)$ \\
\hline & $30-35$ & $31(12.7 \%)$ & $10(4.1 \%)$ & $10(4.1 \%)$ & $11(4.5 \%)$ \\
\hline & $>35$ & $4(1.6 \%)$ & $2(0.8 \%)$ & $2(0.8 \%)$ & $0(0 \%)$ \\
\hline
\end{tabular}

TABLE 2: Distribution maternal risk factors for neonatal sepsis among neonates admitted in Shashemenne referral hospital and Melka Oda district hospital in NICU wards, Ethiopia, 2017.

\begin{tabular}{|c|c|c|c|c|c|}
\hline \multirow{2}{*}{ VARIABLE } & & \multirow{2}{*}{ Frequency } & \multicolumn{3}{|c|}{ Sepsis } \\
\hline & & & EONS & LONS & OTHERS \\
\hline \multirow{2}{*}{ History of maternal UTI } & Yes & $22(9 \%)$ & $7(2.9 \%)$ & $7(2.9 \%)$ & $8(3.4 \%)$ \\
\hline & No & $222(91 \%)$ & $116(47.5 \%)$ & $60(24.6 \%) \%)$ & $46(18.9 \%)$ \\
\hline \multirow{2}{*}{ History of antenatal care } & Yes & $192(78.7 \%)$ & $101(41.4 \%)$ & $52(21.3 \%)$ & $39(16 \%)$ \\
\hline & No & $52(21.3 \%)$ & $22(9 \%)$ & $15(6 \%)$ & $15(6 \%)$ \\
\hline \multirow{2}{*}{ History of Maternal Fever } & Yes & $36(14.8 \%)$ & $14(5.7 \%)$ & $11(4.5 \%)$ & $11(4.5 \%)$ \\
\hline & No & $208(85.2)$ & $109(44.7 \%)$ & $56(23 \%)$ & $43(17.6 \%)$ \\
\hline \multirow{2}{*}{ History of foul smelling liquor } & Yes & $10(4.1 \%)$ & $5(2 \%)$ & $2(0.8 \%)$ & $3(1.2 \%)$ \\
\hline & No & $234(95.9 \%)$ & $118(48.4 \%)$ & $65(26.6 \%)$ & $51(20.9 \%)$ \\
\hline \multirow{2}{*}{ History of Chorioamnionitis } & Yes & $12(4.9 \%)$ & $6(2.5 \%)$ & $3(1.2 \%)$ & $3(1.2 \%)$ \\
\hline & No & $232((95.1 \%)$ & $117(48 \%)$ & $64(26 \%)$ & $51(20.9 \%)$ \\
\hline \multirow{2}{*}{ Meconium stained amniotic fluid } & Yes & $17(7 \%)$ & $7(2.9 \%)$ & $3(1.2 \%)$ & $7(2.9 \%)$ \\
\hline & No & $227(93 \%)$ & $116(47.5 \%)$ & $64(26 \%)$ & $47(19 \%)$ \\
\hline \multirow{2}{*}{ History of premature rupture of membrane } & Yes & $52(21.3 \%)$ & $18(7 \%)$ & $9(3.7 \%)$ & $9(3.7 \%)$ \\
\hline & No & $192(78.7 \%)$ & $100(41 \%)$ & $49(20 \%)$ & $43(17.6 \%)$ \\
\hline \multirow{2}{*}{ Duration of PROM } & $<18 \mathrm{hrs}$ & $174(71.3 \%)$ & $96(39 \%)$ & $42(17.2 \%)$ & $36(14.8 \%)$ \\
\hline & $>18 \mathrm{hrs}$ & $24(9.8 \%)$ & $10(4 \%)$ & $8(3.2 \%)$ & $6(2.5 \%)$ \\
\hline \multirow{2}{*}{ Parity } & Primi & $99(40.6 \%)$ & $52(21.3 \%)$ & $23(9.4 \%)$ & $24(\%)$ \\
\hline & Multipara & $145(59.4 \%)$ & $71(9.8 \%)$ & $44(18 \%)$ & $30(12.3 \%)$ \\
\hline \multirow{3}{*}{ Mode of delivery } & $\mathrm{S} / \mathrm{V}$ & $181(74.2$ & $86(35.2 \%)$ & $53(21.7 \%)$ & $42(17.2 \%)$ \\
\hline & $\mathrm{C} / \mathrm{S}$ & $52(21.3 \%)$ & $32(13 \%)$ & $12(5 \%)$ & $8(3.3 \%)$ \\
\hline & Vacuum(I) & $11(4.5 \%)$ & $5(2 \%)$ & $2(0.8 \%)$ & $4(1.6 \%)$ \\
\hline \multirow{4}{*}{ Place of delivery } & Hospital & $181(75 \%)$ & $98(40 \%)$ & $46(18.9 \%)$ & $39(16 \%)$ \\
\hline & Health Center & $36(14.6 \%)$ & $18(7.3 \%)$ & $9(3.7 \%)$ & $9(3.7 \%)$ \\
\hline & Clinic & $6(2.5 \%)$ & $3(1.2 \%)$ & $2(0.8 \%)$ & $1(0.4 \%)$ \\
\hline & Home & $19(7.8 \%)$ & $4(1.6 \%)$ & $14(5.7 \%)$ & $1(0.4 \%)$ \\
\hline \multirow{4}{*}{ Duration of labor } & $<6 \mathrm{hrs}$ & $31(12.7 \%)$ & $10(4 \%)$ & $13(5.3 \%)$ & $8(3.3 \%)$ \\
\hline & 6-12 hrs & $74(32.6 \%)$ & $39(16 \%)$ & $19(7.8 \%)$ & $16(7 \%)$ \\
\hline & $12-24 \mathrm{hrs}$ & $73(32.1)$ & $41(6.6 \%)$ & $18(7.4 \%)$ & $14(5.7 \%)$ \\
\hline & $>24 \mathrm{hrs}$ & $16(7 \%)$ & $12(5 \%)$ & $0(0 \%)$ & $4(1.6 \%)$ \\
\hline Diagnosis on admission & & & $123(50.4 \%)$ & $67(27.5 \%)$ & $52(22.1 \%)$ \\
\hline
\end{tabular}


TABLE 3: Birth related factors predisposing a neonate for neonatal sepsis among neonates admitted in Shashemenne referral hospital and Melka Oda district hospital in NICU wards, Ethiopia, 2017.

\begin{tabular}{|c|c|c|c|c|c|}
\hline \multirow[b]{2}{*}{ Variable } & & \multicolumn{4}{|c|}{ Sepsis } \\
\hline & & Frequency & EONS & LONS & OTHERS \\
\hline \multirow{3}{*}{ Birth weight } & LBW $<2.5 \mathrm{~kg}$ & $69(28.3 \%)$ & $25(10.2 \%)$ & $21(8.6 \%)$ & $23(9.4 \%)$ \\
\hline & NBW $2.5 \mathrm{~kg}$ & $166(68 \%)$ & $93(38.1 \%)$ & $44(18 \%)$ & $29(11.9 \%)$ \\
\hline & Over, $>4 \mathrm{~kg}$ & $9(3.7 \%)$ & $5(2 \%)$ & $2(.8 \%)$ & $2(0.8 \%)$ \\
\hline \multirow{2}{*}{ Gestational age } & Pre-term <37wks & $65(26.6 \%)$ & $21(8.6 \%)$ & $21(8.6 \%)$ & $23(9.4 \%)$ \\
\hline & Term37-42wks & $179(73.4 \%)$ & $102((41.8)$ & $46(18.9 \%)$ & $31(12.7 \%)$ \\
\hline \multirow{2}{*}{ Birth asphyxia } & Yes & $56(23 \%)$ & $21(8.6 \%)$ & $10(4 \%)$ & $25(10.2 \%)$ \\
\hline & No & $188(77 \%)$ & $102(41.8 \%)$ & $57(23.4 \%)$ & $29(11.9 \%)$ \\
\hline \multirow{2}{*}{ APGAR score } & $>7$ & $132(54.1 \%)$ & $68(27.9 \%)$ & $46(18.9 \%)$ & $18(7.4 \%)$ \\
\hline & $<7$ & $112(45.9 \%$ & $55(22.5 \%)$ & $21(8.6 \%)$ & $36(14.8 \%)$ \\
\hline \multirow{2}{*}{ Outcome } & Died & $46(18.9 \%)$ & $30(12.3 \%)$ & $10(4 \%)$ & $6(2.3 \%)$ \\
\hline & Cured & $198(81.1 \%)$ & $123(50.4 \%)$ & $40(16.4 \%)$ & $35(14.3 \%)$ \\
\hline
\end{tabular}

TABLE 4: Medical risk factors for neonatal sepsis among neonates admitted in Shashemenne referral hospital and Melka Oda district hospital in NICU wards, Ethiopia, 2017.

\begin{tabular}{|c|c|c|c|c|c|}
\hline \multirow{2}{*}{ Variable } & & \multirow{2}{*}{ Frequency } & \multicolumn{3}{|c|}{ Sepsis } \\
\hline & & & EONS & LONS & OTHER \\
\hline \multirow{2}{*}{ Mechanical ventilation } & Yes & $98(40.2 \%)$ & $57(23.4 \%)$ & $13(5 \%)$ & $28(11.4 \%)$ \\
\hline & No & $146(59.8 \%)$ & $66(27 \%)$ & $54(22 \%)$ & $26(10.7 \%)$ \\
\hline \multirow{2}{*}{ Oxygen via nasal catheter } & Yes & $143(58.6 \%)$ & $66(27 \%)$ & $40(16.4 \%)$ & $37(15 \%)$ \\
\hline & No & $101(41.4 \%)$ & $57(23.4 \%)$ & $27(11 \%)$ & $17(7 \%)$ \\
\hline \multirow{2}{*}{ Oxygen via mask } & Yes & $47(19 \%)$ & $16(6.6 \%)$ & $10(4 \%)$ & $21(8.6 \%)$ \\
\hline & No & $197(81 \%)$ & $107(43.9 \%)$ & $57(23.4 \%)$ & $33(13.5 \%)$ \\
\hline \multirow{2}{*}{ Suspected neonatal sepsis And other cases } & & $190(77.9 \%)$ & & & \\
\hline & & $54(22.1 \%)$ & & & \\
\hline
\end{tabular}

NB: $\mathrm{P}<0.05$, CI- $95 \%$ and $*$ had association.

had developed neonatal sepsis. Referring to APGAR score about $132(54.1 \%)$ neonates had APGAR score $>7$ and out of them 114 (59\%) of them developed sepsis. About 112 (45.9\%) neonates had APGAR score less than 7 and 76 (31.1\%) of them were presented with neonatal sepsis (Table 3 ).

3.4. Medical Risk Factors of Neonatal Sepsis. From the total 244 study units $98(40.2 \%)$ neonates were reported to have mechanical ventilation; out of this 70 (28.7\%) of them had neonatal sepsis. Among the total study population more than three-fourth of them 143 (58.6\%) had oxygen via catheter and $106(74.1 \%)$ of these neonates developed neonatal sepsis (Table 4).

3.5. Factors Associated with Neonatal Sepsis. On this study, bivariate logistic regression analysis revealed that age of neonates, maternal history of meconium aspiration, birth asphyxia, use of oxygen via mask, low birth weight, and gestational age less than 37 weeks show significant association with neonatal sepsis. Multivariate logistic regression neonates whose age were less than seven days were 3 times more likely to develop neonatal sepsis compared with the age of neonates greater than eight days of age $(\mathrm{AOR}=3.01$ with $95 \% \mathrm{CI}$ $(1.148,7.89)$. Neonates who had birth asphyxia were 3.54 times more likely to have neonatal sepsis compared to those who did not have birth asphyxia (AOR=3.54 95\%CI (1.57,7.984)). Finally the neonates who used oxygen via mask were 2.859 times highly at risk to develop neonatal sepsis compared to neonates those who did not used it at birth $[\mathrm{AOR}=2.859$ with 95\%CI $(1.300,6.289)]$ (Table 5).

\section{Discussion}

In this study the overall prevalence of neonatal sepsis was $77.9 \%$. The study conducted in Iran shows that prevalence of neonatal sepsis was $51.8 \%$ [10]. This difference could be due to smaller sample size and difference in sociodemographic status of population as well as accessibility of health facility and differences in the definition of neonatal sepsis among the studies. Although other studies in Egypt reported that prevalence of neonatal sepsis was $40.7 \%$ [11], this difference could be due to the fact that they use confirmed laboratory results based on blood culture. Although this study finding is greater than the study conducted in Black Lion specialized hospital in 2010 which was $44.7 \%$ [12], this difference might be because culture is not used for diagnosing neonatal sepsis in this study and clinical features alone were used in both hospitals. In addition, the other possible reason might be that 
TABLE 5: Bivariate and multivariate analysis showing the association between neonatal sepsis and others different variables in neonates admitted to Shashemene governmental hospital, NICU, Ethiopia, 2017, GC.

\begin{tabular}{|c|c|c|c|c|c|c|}
\hline \multirow{2}{*}{\multicolumn{2}{|c|}{ Variable }} & \multicolumn{5}{|c|}{ Neonatal sepsis } \\
\hline & & Yes & No & $P$ value & COR with 95\%CI & AOR with 95\% CI \\
\hline \multirow{2}{*}{ Age of neonates } & 0-7 days & $113(46.3 \%)$ & $68(27.8 \%)$ & $.025 *$ & $2,806(1.196-6.585) *$ & $3.01(1.148-7.89)$ \\
\hline & 8-28 days & $46(18.8 \%)$ & $17(7 \%)$ & & 1 & 1 \\
\hline \multirow{2}{*}{ MSAF } & Yes & $10(4 \%)$ & $180(73.8 \%)$ & .177 & $2.979(1.054-8.416)$ & $2.223(.697-7.087)$ \\
\hline & No & $7(29 \%)$ & $47(19.3 \%)$ & & 1 & 1 \\
\hline \multirow{2}{*}{ BW } & LBW & $46(18.9 \%)$ & $23(9.4 \%)$ & .453 & $1.541(1.233-4.376)$ & $2.302(.261-20.329)$ \\
\hline & NW & $144(59 \%)$ & $31(12.7 \%)$ & & 1 & 1 \\
\hline \multirow{2}{*}{ GA } & Preterm & $46(18.9 \%)$ & $23(9.4 \%)$ & .277 & $2.678(1.411-5.083)$ & $2.167(.537-8.745)$ \\
\hline & Term & $148(60.7 \%)$ & $31(12.7 \%)$ & & 1 & 1 \\
\hline \multirow{2}{*}{ BA } & Yes & $31(12.7 \%)$ & $25(10.2 \%)$ & $.000 *$ & $4.422(2.288-8.546)$ & $3.540(1.570-7.984)$ \\
\hline & No & $159(65.2 \%)$ & $29(11.9 \%)$ & & 1 & 1 \\
\hline \multirow{2}{*}{ MV } & Yes & $70(28.7 \%)$ & $28(11.5 \%)$ & .288 & $1.873(1.017-3.448)$ & $1.466(.724-2.969)$ \\
\hline & No & $120(49.2 \%)$ & $26(10.7 \%)$ & & 1 & 1 \\
\hline \multirow{2}{*}{ OVM } & Yes & $25(10.2 \%)$ & $21(8.6 \%)$ & $.009 *$ & $4.348(2.171-8.712)$ & $2.859(1.300-6.289)$ \\
\hline & No & $164(67.2 \%)$ & $33(13.5 \%)$ & & 1 & 1 \\
\hline
\end{tabular}

the area and the community are far from the center of the country and from health facilities which in turn decreases of the population and increases risk of harmful traditional practices on children. There was another study conducted in Douala Cameroon in urban district hospital in 2014 which reported that the prevalence of NS was 79.1\% [13].

\section{Conclusion}

In the study we found that among a total of neonates admitted in Shashemene hospital, the overall prevalence of neonatal sepsis by physicians diagnosis from the clients medical record was $77.9 \%$ within the last one year from two hospitals. The factors associated with neonatal sepsis were birth asphyxia, age being less than 7 days, and use of oxygen via mask.

\section{Abbreviations}

ART: Antiretroviral treatment

ANC: Antenatal care

AOR: Adjusted Odds Ratio

APGAR: Activity, pulse, Grimace, appearance, and respiration

BA: Birth asphyxia

CI: Confidence interval

COR: Crude Odds Ratio

EDHS: Ethiopia Demographic and Health Survey

EONS: Early onset neonatal sepsis

LBW: Low birth weight

LONS: Late onset neonatal sepsis

MCH: Maternal to child health

MDG: Millennium development goal

MSAF: Meconium stained amniotic fluid

NICU: Neonatal intensive care unit

NMR: Neonatal mortality rate

OPD: Outpatient department
OVM: Oxygen via mask

PROM: Prolonged rupture of membrane

SPSS: Statistical Package for Social Science

TB: Tuberculosis

TTBA: Traditional trained birth attendant

UTI: Urinary tract infection

WHO: World Health Organization.

\section{Data Availability}

The datasets used and/or analyzed during the current study are available for ethical reasons from the corresponding author and coauthors.

\section{Ethical Approval}

The study protocol was ethically approved by the Ethical Review Board (IRB) of Ambo University College of Medicine and Health Sciences. An official letter was written to Shashemene town health office.

\section{Consent}

The study posed a low or not more than a minimal risk to the study participants. Also, the study did not involve any invasive procedures. Accordingly, after the objective of the study was explained, verbal consent was obtained from the respondents of the study. Moreover, the confidentiality of information was guaranteed by using code numbers rather than personal identifiers and by keeping the data locked.

\section{Conflicts of Interest}

The authors declare that they have no conflicts of interest. 


\section{Authors' Contributions}

Aytenew Getabelew and Mihret Aman conceived and designed the study and analyzed the data. Tomas Yeheyis prepared the manuscript. Endashaw Fantaye and Tomas Yeheyis assisted with the design conception and critically reviewed the manuscript. All authors have read and approved this manuscript.

\section{Acknowledgments}

The authors would like to express their gratitude to Ambo University Faculty of Medicine and Health Science, Department of Nursing, for giving them the opportunity to do this research paper. Special thanks are also due to their advisors Mr. Endashaw Fantaye (B.S., M.S.) for his unreserved encouragement and provision of constructive comments and guidance in the development of this research paper. Also their deepest gratitude goes to Shashemene hospital administrative and staff members, especially those who work in the card room for their participation and unreserved support for the success of this study. Finally they are very grateful to their wonderful family for their prayers and for giving them the peace of mind to be able to undertake this research work to a successful end. Last but not least, they extend their thanks to their friends who contribute directly and indirectly from the beginning up to now for the development of this research.

\section{References}

[1] A. K. M. Zaidi, H. A. Ganatra, S. Syed et al., "Effect of case management on neonatal mortality due to sepsis and pneumonia," BMC Public Health, vol. 11, no. 3, article no. S13, 2011.

[2] C. Chiesa, A. Panero, J. F. Osborn, A. F. Simonetti, and L. Pacifico, "Diagnosis of Neonatal Sepsis: A Clinical and Laboratory Challenge," Clinical Chemistry, vol. 50, no. 2, pp. 279-287, 2004.

[3] G. Yamey, H. Horváth, L. Schmidt, J. Myers, and C. D. Brindis, "Reducing the global burden of Preterm Birth through knowledge transfer and exchange: A research agenda for engaging effectively with policymakers," Reproductive Health, vol. 13, no. 1, article no. 26, 2016.

[4] K. Wechselberger, A. Schmid, A. Posod et al., "Secretoneurin serum levels in healthy term neonates and neonates with hypoxic-ischaemic encephalopathy," Neonatology, vol. 110, no. 1, pp. 14-20, 2016.

[5] N. Aijaz, N. Huda, and S. Kausar, Disease Burden of NICU, vol. 6, Tertiary Care Hospital, Karachi, Pakistan, 2012.

[6] M. F. Health Sector Transformation Plan, 015/16 - 2019/20 (2008-2012n EFY) Draft_V2. feb2015.

[7] Central statisticalAgency (CSA) and Ethiopian Demographic health survey Addis Ababa Ethiopia, 2016.

[8] J. E. Lawn, S. Cousens, and J. Zupan, “4 Million neonatal deaths: when? Where? Why?, The Lancet, vol. 365, no. 9462, pp. 891900, 2005.

[9] D. Gebremedhin, H. Berhe, and K. Gebrekirstos, "Risk factors for neonatal sepsis in public hospitals of Mekelle City, North Ethiopia, 2015: Unmatched case control study," PLOS ONE, vol. 11, no. 5, Article ID e0154798, 2016.
[10] M. Rakhsha, L. Pourali, S. Ayati et al., "Effective Maternal and Neonatal Factors Associated with the Prognosis of Preterm Infants in Iran Patient SafQualImprov," 2016.

[11] E. M. R. Shehab El-Din, M. M. A. El-Sokkary, M. R. Bassiouny, and R. Hassan, "Epidemiology of neonatal sepsis and implicated pathogens: A Study from Egypt," BioMed Research International, vol. 2015, Article ID 509484, 2015.

[12] D. Shitaye, D. Asrat, Y. Woldeamanuel, and B. Worku, "Risk factors and etiology of neonatal sepsis in Tikur Anbessa University Hospital, Ethiopia.," Ethiopian Medical Journal, vol. 48, no. 1, pp. 11-21, 2010.

[13] E. Mah-Mungyeh, A. Chiabi, F. L. Tchokoteu et al., "Neonatal mortality in a referral hospital in Cameroon over a seven year period: Trends, associated factors and causes," African Health Sciences, vol. 14, no. 4, pp. 985-992, 2014. 


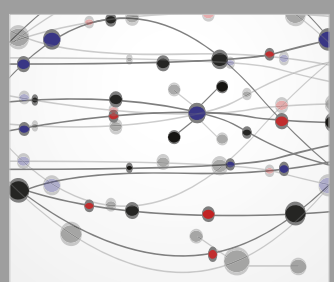

The Scientific World Journal
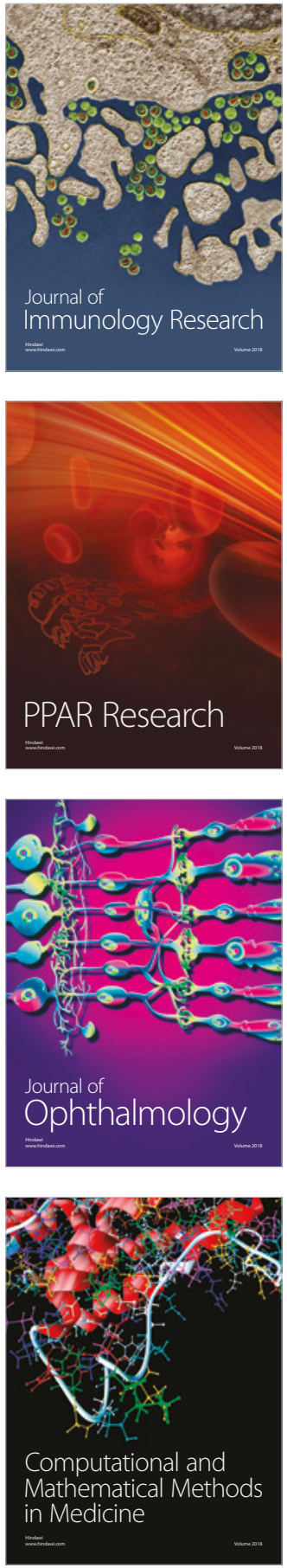

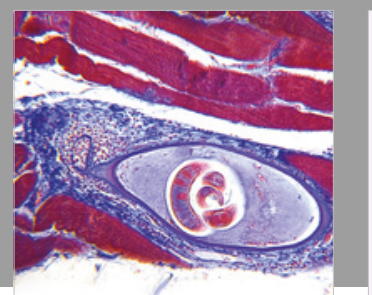

Gastroenterology Research and Practice

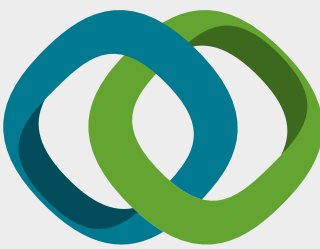

\section{Hindawi}

Submit your manuscripts at

www.hindawi.com
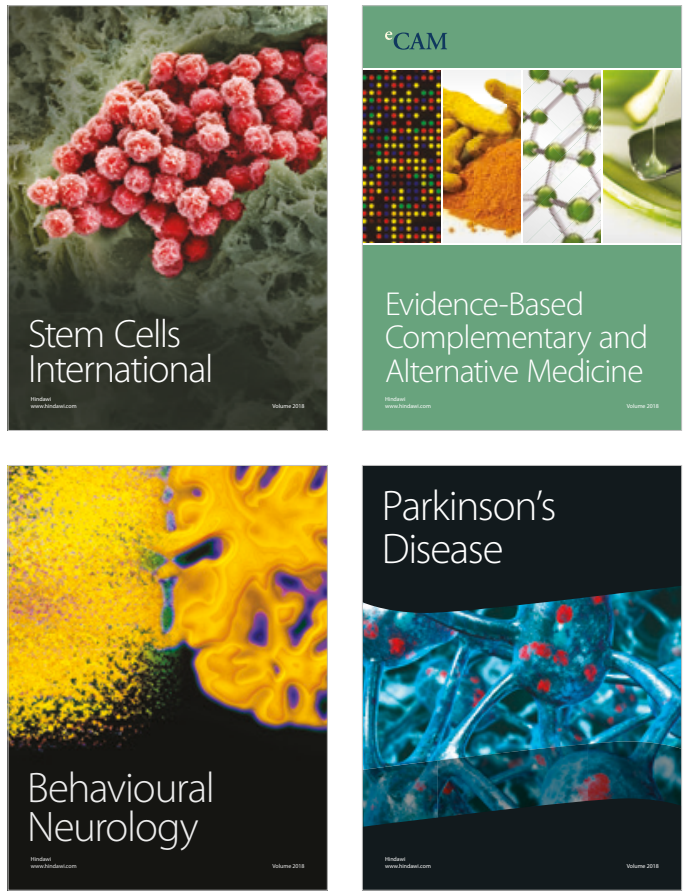

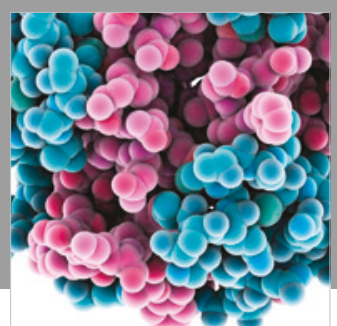

ournal of

Diabetes Research

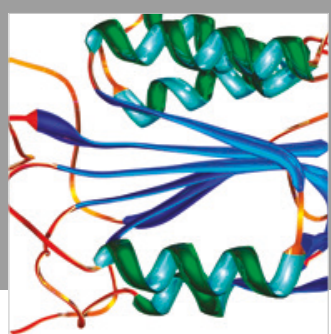

Disease Markers
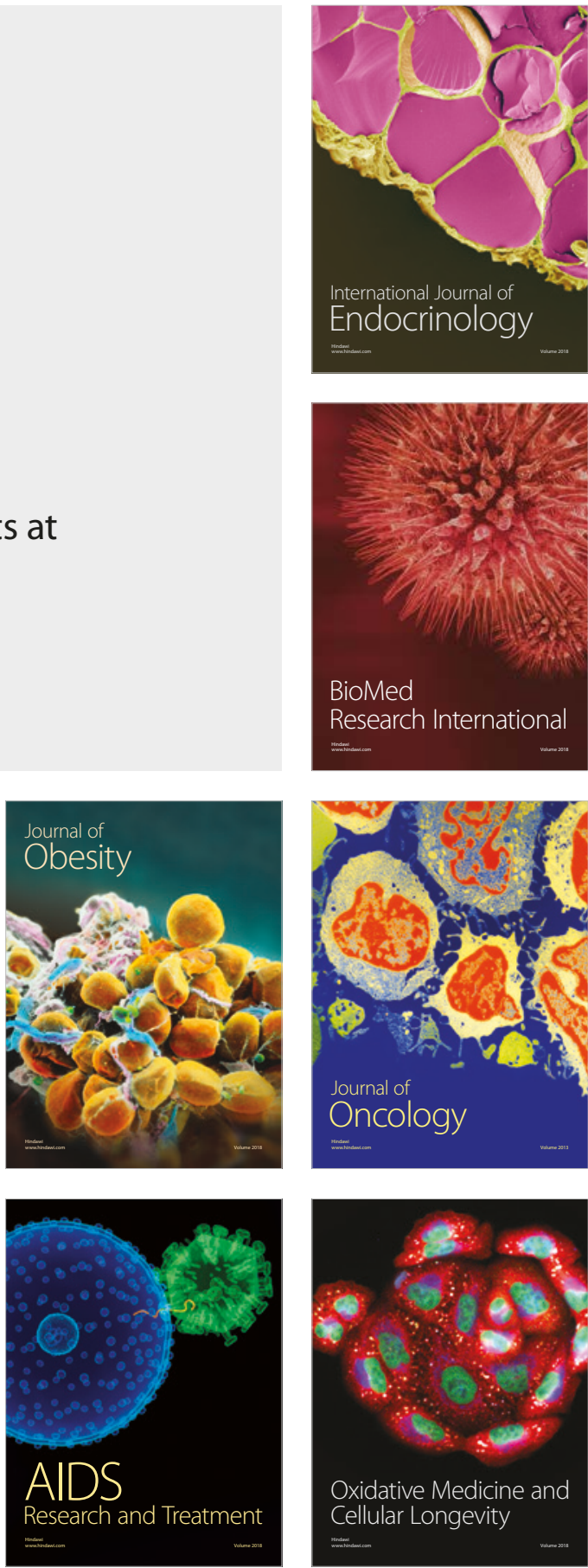